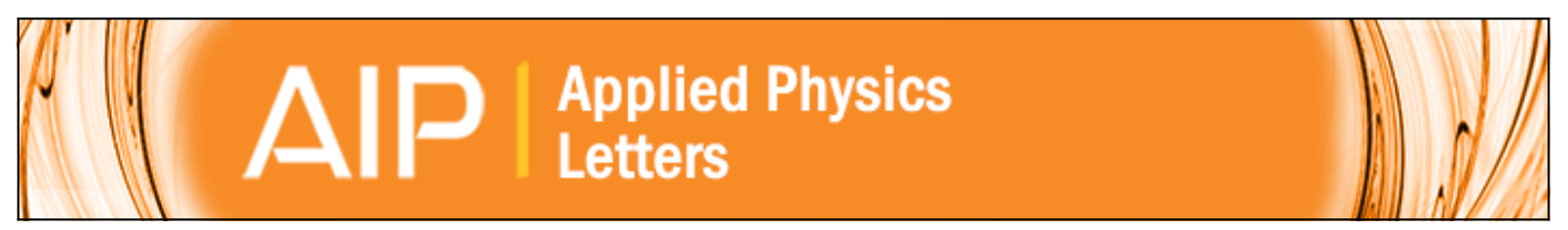

\title{
Phonon transport in an initially twisted polyvinyl acetate nanofiber
}

Monrudee Liangruksa and Ishwar K. Puri

Citation: Applied Physics Letters 102, 191907 (2013); doi: 10.1063/1.4804428

View online: $h t t p: / / d x . d o i . o r g / 10.1063 / 1.4804428$

View Table of Contents: http://scitation.aip.org/content/aip/journal/apl/102/19?ver=pdfcov

Published by the AIP Publishing

\section{AlP Re-register for Table of Content Alerts}




\title{
Phonon transport in an initially twisted polyvinyl acetate nanofiber
}

\author{
Monrudee Liangruksa ${ }^{1, a)}$ and Ishwar K. Puri ${ }^{2}$ \\ ${ }^{1}$ National Nanotechnology Center, National Science and Technology Development Agency, \\ 111 Thailand Science Park, Phahonyothin Road, Pathumthani 12120, Thailand \\ ${ }^{2}$ Department of Engineering Science and Mechanics, Virginia Tech, Blacksburg, Virginia 24061, USA
}

(Received 20 March 2013; accepted 23 April 2013; published online 14 May 2013)

\begin{abstract}
A reduction in phonon thermal conductivity enhances thermoelectric performance. One method to accomplish this is by applying mechanical stress to a nanostructure. We consider an initially twisted $20 \mathrm{~nm}$ polyvinyl acetate nanofiber undergoing torsion. Our analytical method uses the continuum approach of Mooney's model. Torsion modifies the phonon dispersion. Angles of twist between $45^{\circ}$ and $70^{\circ}$ reduce the averaged phonon group velocity and the phonon thermal conductivity but when these angles are increased further, this conductivity increases. This suggests a phonon engineering approach to tune the thermal conductivity of nanomaterials. (C) 2013 AIP Publishing LLC. [http://dx.doi.org/10.1063/1.4804428]
\end{abstract}

The efficiency of a thermoelectric device is represented in terms of its figure of merit $Z T=S^{2} G T / \kappa$. Here, $T$ denotes temperature, $S$ is the Seebeck coefficient, and $G$ is electrical conductivity. The thermal conductivity $\kappa=\kappa_{e}+\kappa_{l}$, where $\kappa_{e}$ and $\kappa_{l}$, respectively, denote the conductivities of the electrons that are transported through the material and of the phonons travelling through the lattice. ${ }^{1-3}$ The Seebeck coefficient incorporates diffusive effects, which occur through the spatial redistribution of the charge carriers as well as the phonon drag that is produced due to the interaction of these carriers with the nonequilibrium phonon distribution. The drag is generally neglected when the interfacial scattering of phonons is relatively high, since scattering has a stronger influence on the phonon mean free path than the drag. The thermoelectric power attributable to the phonon drag is in any case small for a heavily doped material as compared to charge carrier diffusion. ${ }^{4}$

Low dimensional systems can enhance $Z T$ by directionally confining the motion of electrons and holes, thus, changing the shape of the electronic density of states (DOS). ${ }^{5}$ The DOS refers to the number of different states per unit volume that electrons can occupy at a particular energy level. It has a delta function shape for onedimensional (1D) nanostructures, a step function form for two-dimensional (2D) nanostructures, and a parabolic distribution for bulk materials. A delta form for the DOS implies that the electronic states are enhanced at the Fermi level, thus, increasing $S .^{6}$

The figure of merit can also be improved by reducing the thermal conductivity of a thermoelectric material. For most semiconductors and insulators that experience low electron conduction, the major contribution to the thermal conductivity arises from acoustic phonons, which are quanta of lattice vibrations. Experiments have established that nanostructures decrease the thermal conductivity ${ }^{13-17}$ since phonon scattering at the boundary is enhanced as the material dimension becomes smaller. This increase in scattering

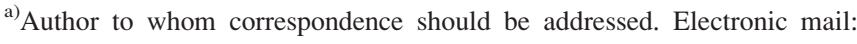
monrudee@nanotec.or.th
}

decreases the phonon mean free path and consequently the thermal conductivity.

Solid materials such as Bismuth-Telluride $\left(\mathrm{Bi}_{2} \mathrm{Te}_{3}\right)$, Antimony-Telluride $\left(\mathrm{Sb}_{2} \mathrm{Te}_{3}\right)$, and Silicon-Germanium ( $\mathrm{SiGe}$ ) are used for thermoelectric applications. Due to its relatively large $Z T, \mathrm{Bi}_{2} \mathrm{Te}_{3}$ is used in Peltier coolers. However, it is toxic and Tellurium is a scarce element. ${ }^{7}$ Conductive polymers, which can be doped to improve their electrical conductivity, can be suitable alternatives. Compared to inorganic materials, they are easier to process, are cheaper, have tunable physical and chemical properties, as well as a low thermal conductivity. Thermoelectric polymers include polyacetylene, ${ }^{8,9}$ polypyrroles,${ }^{10,11}$ polyanilines, ${ }^{11,12}$ polythiophenes, ${ }^{13}$ and poly $(2,7$-carbazole $) .{ }^{14}$ The conductive polymer PEDOT $^{15-17}$ poly(3,4-ethylenedioxythiophene) is widely used in high conductivity coatings, organic LED displays, ${ }^{18}$ nanofiber electrodes, ${ }^{19}$ and solar cells. Since typical polymers do not have a high enough electrical conductivity for thermoelectric applications, polymer nanocomposites with improved electrical conductivity have been proposed as a potential solution. ${ }^{20-23}$ Polymers, such as polyvinyl acetate (PVAc), can be mixed with thermoelectric particles to enhance thermoelectric performance. ${ }^{24}$ Such a material can have an electrical conductivity comparable to that of carbon nanotubes but with a thermal conductivity near that of its polymer matrix. ${ }^{24}$ During fabrication, it is inevitable that conductive polymers are bent, twisted, stretched, and compressed, i.e., they do not retain straight shapes. These helical or twisted shapes can have their own interesting properties and promising applications.

Phonon transport is modified by the geometry (since it alters phonon confinement), composition, and temperature of a nanomaterial. Nanoscale confinement effects change the phonon dispersion and the phonon group velocity, producing a thermal conductivity that is different from that of the bulk material. Numerous investigations have addressed the effects of phonon confinement on $Z T$ in nanostructures, ${ }^{3,25-30}$ but there is still a paucity of literature related to the effects of phonon tailoring by mechanical stresses. $^{31}$ 
Here, we investigate phonon transport in an initially twisted nanofiber composed of PVAc by modifying the wave equations that are based on Mooney's model by accounting for the angles of twist. Then, we calculate the phonon dispersion, the phonon group wave velocity, and the thermal conductivity for different twist angles. The results show that torsion modifies phonon dispersion and point to potential thermoelectric applications for twisted nanofibers.

We consider a circular cylinder made of Mooney material of infinite length and radius $a$. The cylinder is subjected to a pre-torsion characterized by the angle of twist $K$. The Mooney model is valid for rubberlike materials that are isotropic and incompressible, and undergo large elastic deformations. The stress tensor due to this deformation is a solution of Cauchy equations in the deformed state, $^{32}$

$$
{ }_{0} t_{; l}^{k l}=0
$$

For an incompressible material, the stress tensor can be expressed in terms of the deformation field through the constitutive relations,

$$
{ }_{0} t^{k l}={ }_{0} \Phi_{0}^{-1} c^{k l}+{ }_{0} P_{0} g^{k l}+{ }_{0} \Psi_{0} B^{k l},
$$

where

$$
\begin{aligned}
{ }_{0} B^{k l} & ={ }_{0} I_{1} \cdot{ }_{0}^{-1} c^{k l}-{ }_{0}^{-1} c^{k m} \cdot{ }_{0}^{-1} c^{m l},{ }_{0} \Phi=22_{0}\left(\partial \Sigma / \partial I_{1}\right), \\
{ }_{0} \Psi & =2_{0}\left(\partial \Sigma / \partial I_{2}\right) .
\end{aligned}
$$

Here, $\Sigma$ denotes the stress potential, ${ }_{0} I_{1},{ }_{0} I_{2}$, and ${ }_{0} I_{3}$ $=1$ are the invariants of ${ }_{0}^{-1} c,{ }_{0} g^{k l}$ is the reciprocal metric tensor in the deformed region, and ${ }_{0} P$ is a function of coordinates determined through the field equations and boundary conditions. An infinitely small displacement field $\mathbf{u}(\mathbf{x}, t)$ is superimposed on the specified initial deformation. We denote the increment in the stress tensor as $\mathbf{t}(\mathbf{x}, t)$. Hence, the equations including the superimposed motion and constitutive relation are

$$
\begin{gathered}
t_{; l}^{k l}+\left\{\begin{array}{c}
k \\
l m
\end{array}\right\} t^{l m}+\left\{\begin{array}{c}
l \\
l m
\end{array}\right\} 0 t^{k m}=\rho \partial^{2} u^{k} / \partial t^{2}, \text { and } \\
t^{k l}=\Phi_{0}^{-1} c^{k l}+{ }_{0} P g^{k l}+p_{0} g^{k l}+{ }_{0} \Psi B^{k l}+\Psi_{0} B^{k l},
\end{gathered}
$$

where

$$
\begin{aligned}
& g_{k l}=u_{k ; l}+u_{l ; k}, g^{k l}=-{ }_{0} g^{k m}{ }_{0} g^{\ln } g_{m n},\left\{\begin{array}{c}
l \\
m k
\end{array}\right\}=1 / 2{ }_{o} g^{\ln }\left(g_{m n, k}+g_{k n, m}-g_{m k, n}\right)+1 / 2 g^{\ln }\left(0 g_{m n, k}+0 g_{k n, m}-{ }_{0} g_{m k, n}\right)=u_{; m k}^{l}, \\
& I_{1}={ }_{0}^{-1} c^{k m} g_{m k}, I_{2}={ }_{0} B^{l m} g_{m l}, B^{k l}=I_{1}{ }_{0}^{-1} c^{k l}-{ }_{0}^{-1} c^{k m} g_{m n}{ }_{0}^{-1} c^{n l}, \Phi=A I_{1}+F I_{2}, \Psi=F I_{1}+B I_{2}, A=2_{0}\left(\partial^{2} \Sigma / \partial I_{1}^{2}\right), \\
& B=2_{0}\left(\partial^{2} \Sigma / \partial I_{2}^{2}\right), \text { and } F=2_{0}\left(\partial^{2} \Sigma / \partial I_{1} \partial I_{2}\right) .
\end{aligned}
$$

The symbol $\rho$ denotes the density of the medium and $p$ is an unknown function of coordinates and time. For incompressible materials, the displacement field must obey

$$
\vec{\nabla} \cdot \vec{u}=0 .
$$

For a rubberlike incompressible material, the stress potential proposed by Mooney,

$$
\Sigma=\alpha\left(I_{1}-3\right)+\beta\left(I_{2}-3\right),
$$

where $\alpha$ and $\beta$ are positive constants that depend upon the material. Based on Eq. (3),

$$
{ }_{0} \Phi=2 \alpha,{ }_{0} \Psi=2 \beta,
$$

and from Eq. (5),

$$
A=B=F=0, \Phi=\Psi=0 .
$$

Thus, the constitutive relation of Eq. (4) can be simplified based on the Mooney material assumption, i.e.,

$$
t^{k l}={ }_{0} P g^{k l}+p_{o} g^{k l}+2 \beta B^{k l} .
$$

Considering that the surface of the cylinder is free from traction, the stress field in cylindrical coordinates,

$$
\begin{aligned}
& { }_{0} t^{r r}=\alpha K^{2}\left(r^{2}-a^{2}\right),{ }_{0} t^{\theta \theta}=\alpha K^{2} / r^{2}\left(3 a^{2}-r^{2}\right), \\
& { }_{0} t^{\theta z}=2(\alpha+\beta) K \text { and }{ }_{0} t^{z z}=-2 K^{2}\left[\alpha\left(a^{2}-r^{2}\right)+\beta r^{2}\right], \\
& { }_{0} t^{r \theta}={ }_{0} t^{t^{z}}=0 .
\end{aligned}
$$

The hydrostatic pressure,

$$
{ }_{0} P=-2 \beta K^{2} r^{2}+\alpha K^{2}\left(r^{2}-a^{2}\right)-2(\alpha+2 \beta) .
$$

The boundary conditions are

$$
t^{r r}=t^{r \theta}=t^{r z}=0 \quad \text { at } \quad r=a .
$$

The constitutive relations can be simplified into the form: 


$$
\begin{aligned}
t^{r r} & =2\left[2 \alpha+2 \beta+2 \beta K^{2} r^{2}-\alpha K^{2}\left(r^{2}-a^{2}\right)\right] \partial u / \partial r+4 \beta K^{2} r u+4 \beta K r \partial v / \partial z+p, \\
t^{r \theta} & =-\left[2 \alpha+2 \beta-\alpha K^{2}\left(r^{2}-a^{2}\right)\right] v / r^{2}+\left[2 \alpha+2 \beta-\alpha K^{2}\left(r^{2}-a^{2}\right)\right] 1 / r(\partial v / \partial r)-2 \beta K \partial u / \partial z-2 \beta K \partial w / \partial r, \\
t^{r z} & =\left[2 \alpha+2 \beta-\alpha K^{2}\left(r^{2}-a^{2}\right)+2 \beta K^{2} r^{2}\right] \partial u / \partial z+\left[2 \alpha+2 \beta-\alpha K^{2}\left(r^{2}-a^{2}\right)+2 \beta K^{2} r^{2}\right] \partial w / \partial r-2 \beta K r \partial v / \partial r-2 \beta K v, \\
t^{\theta \theta} & =2\left[2 \alpha+2 \beta-\alpha K^{2}\left(r^{2}-a^{2}\right)+2 \beta K^{2} r^{2}\right] u / r^{3}+4 \beta K^{2} \partial u / \partial r+p / r^{2}, \\
t^{\theta z} & =\left[2 \alpha+2 \beta-\alpha K^{2}\left(r^{2}-a^{2}\right)+2 \beta K^{2} r^{2}\right] 1 / r \partial v / \partial z+4 \beta K \partial u / \partial r, \text { and } \\
t^{z z} & =2\left[2 \alpha+2 \beta-\alpha K^{2}\left(r^{2}-a^{2}\right)+2 \beta K^{2} r^{2}\right] \partial w / \partial z+p,
\end{aligned}
$$

where $u(r, z, t), v(r, z, t)$, and $w(r, z, t)$ are the components of displacement vector in the $r-, \theta-$, and $z-$ directions, respectively. Substituting Eq. (14) into Eq. (4), solving for $\left\{\begin{array}{c}k \\ l m\end{array}\right\}$ from Eq. (5) and considering Eq. (6), we obtain a set of linear differential equations that are subjected to the boundary conditions in Eq. (13). We consider harmonic waves of the form:

$$
\left[\begin{array}{c}
u \\
v \\
w \\
p
\end{array}\right]\left[\begin{array}{c}
\bar{u}(r) \\
\bar{v}(r) \\
\bar{w}(r) \\
\bar{p}(r)
\end{array}\right] \operatorname{expi}(q z+\Omega t),
$$

where $\Omega$ and $q$ denote the angular frequency and the wave number of the waves, respectively. This set of linear differential equations is solved to obtain the phonon dispersion relation.

Following Walton ${ }^{33}$ and Saleh and Dubey, ${ }^{34}$ the lattice thermal conductivity of a noncrystalline polymer is a sum of three contributions, i.e., $\kappa=\kappa_{B E}+\kappa_{E M}+\kappa_{A P}$. The $\kappa_{B E}$ contribution arises from phonons interacting with the crystal boundaries, $\kappa_{E M}$ occurs due to empty space scatter, and $\kappa_{A P}$ is attributed to phonons that have frequencies larger than the plateau frequency. Walton proposed that a noncrystalline structure has a fraction of its volume empty. These empty spaces are responsible for the scattering of phonons for which the mean free paths,

$$
\begin{aligned}
& L_{1}^{-1}=(p q) /(4(1-p))+A q^{4} V_{0}, q V_{0}^{1 / 3} \leq 1, \omega<\omega_{p t}, \text { and } \\
& L_{2}^{-1}=B V_{0}^{-1 / 3}, q V_{0}^{1 / 3}>1, \omega>\omega_{p t},
\end{aligned}
$$

where $p$ denotes the fraction of the empty spaces, $q$ is the phonon wave vector, $A$ and $B$ are constants, $V_{O}$ is the critical volume, and $\omega_{p t}$ denotes the plateau frequency corresponding to the plateau temperature. The term $(p q) /(4(1-p))$ describes phonon scattering by empty spaces and the $A q^{4} V_{0}$ is the Rayleigh scattering. The combined scattering relaxation rate is

$$
\begin{aligned}
& \tau_{B E}^{-1}=\tau_{B}^{-1}+a x T+b x^{4} T^{4}, \quad 0<\omega<\omega_{1}, \\
& \tau_{E M}^{-1}=a x T+b x^{4} T^{4}, \quad \omega_{1}<\omega<\omega_{p t}, \text { and } \\
& \tau_{A P}^{-1}=b^{\prime}, \quad \omega_{p t}<\omega<\omega_{D},
\end{aligned}
$$

where $\tau_{B}^{-1}=\left(V_{g}\right)_{a v g} / L$ denotes the boundary scattering relaxation rate due to the Casimir effect. Here, $\left(V_{g}\right)_{\text {avg }}$ denotes the average phonon velocity, $L$ is the Casimir length of the crystal, and $a, b$, and $b^{\prime}$ are constants that can be calculated from the relations $a=0.25 p /(1-p)\left(k_{B} / \hbar\right)$, $b=\left(A V_{0} /\left(V_{g}\right)_{\text {avg }}{ }^{3}\right)\left(k_{B} / \hbar\right)^{4}$, and $b^{\prime}=B\left(V_{g}\right)_{\text {avg }} V_{0}^{-1 / 3}$. In addition, $\omega_{D}$ denotes the Debye frequency, $k_{B}$ is the Boltzmann constant, and $\hbar$ is Planck's constant divided by $2 \pi$. The combined scattering relaxation rate is a sum of three contributions, i.e.,

$$
\begin{gathered}
\kappa_{B E}=C \int_{0}^{T_{1} / T} \tau_{B E} x^{4} e^{x}\left(e^{x}-1\right)^{-2} d x, \\
\kappa_{E M}=C \int_{T_{1} / T}^{T_{2} / T} \tau_{E M} x^{4} e^{x}\left(e^{x}-1\right)^{-2} d x, \text { and } \\
\kappa_{A P}=C \int_{T_{2} / T}^{\theta_{D} / T} \tau_{A P} x^{4} e^{x}\left(e^{x}-1\right)^{-2} d x,
\end{gathered}
$$

where $C=\left(k_{B} / 2 \pi^{2}\left(V_{g}\right)_{a v g}\right)\left(k_{B} T^{3} / \hbar\right)^{3}, \quad T_{1}=\left(\hbar \omega_{1} / k_{B}\right)$, $T_{2}=\left(\hbar \omega_{p t} / k_{B}\right)$, and $\theta_{D}$ is the Debye temperature.

To better understand phonon transport inside a twisted PVAc nanofiber, we consider it to be circular cylinders made of a Mooney material of infinite length and a radius of $10 \mathrm{~nm}$. Such a cylinder is subjected to a pre-torsion characterized by angles of twist $K=0^{\circ}, 30^{\circ}, 36^{\circ}, 45^{\circ}, 60^{\circ}$, and $70^{\circ}$. Here, $K$ is defined as the intersection angle between the planes before and after the cylinder is twisted as shown schematically in Fig. 1. The surface of the cylinder is assumed to be free from traction and the stress field is represented by Eq. (11). The stress potential is approximated by Eq. (7) for purpose of simplification so that the constitutive equations can be written as Eq. (14). These relations are substituted

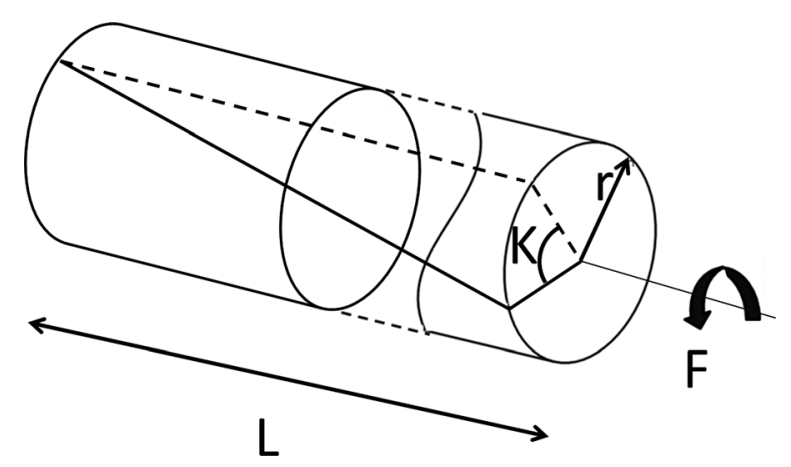

FIG. 1. Schematic showing a twisted PVAc nanofiber of radius $r$. The angle of twist $K$ is defined by the intersection angle between the planes before and after the nanofiber is twisted. 


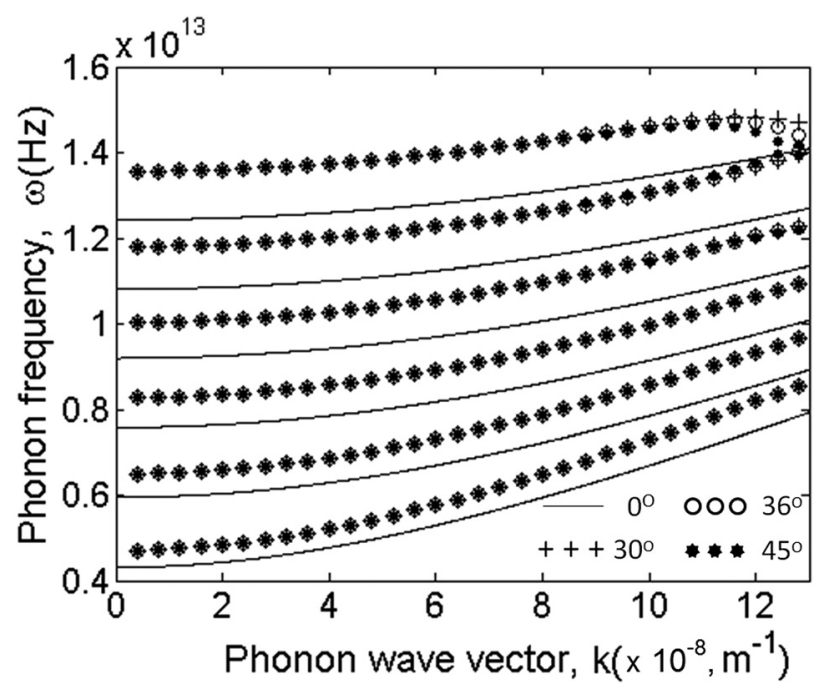

FIG. 2. Phonon dispersion relation for a $20 \mathrm{~nm}$ diameter PVAc under twisting versus the free nanofiber where the angles of twist $K=0^{\circ}, 30^{\circ}, 36^{\circ}$, and $45^{\circ}$.

into the governing equations of Eq. (4) and subject to the traction-free surface boundary conditions.

Solving the governing equations and utilizing parameters from Table I, the dispersion relation for phonons is obtained for PVAc nanofibers at different angles of twist, as shown in Fig. 2. The dispersion for an untwisted nanofiber is different from that for its twisted counterpart in that each mode displays lower energies. At longer wavelengths (smaller $k$ ), phonons for both cases have the same slope, i.e., their velocities are equal. In other words, phonons in an untwisted nanofiber use less energy to travel at the same speed than do phonons in a twisted fiber. For a twisted nanofiber, there is a frequency drop for modes higher than the 4th mode for short wavelength phonons but there is no such change for smaller wave vectors (or longer wavelengths). Hence, short-wavelength phonons are readily scattered by twisting, resulting in changes in the phonon frequency for different angles of twist. The larger the magnitude of twisting the lower is the phonon frequency.

Figure 3 reveals the averaged phonon group wave velocity over angles of twist between $0^{\circ}$ and $45^{\circ}$ is $\left(V_{g}\right)(\omega)=\left[\sum_{n} V_{n} \exp \left(-n \hbar \omega / k_{B} T\right)\right] /\left[\sum_{n} \exp \left(-n \hbar \omega / k_{B} T\right)\right]$, where the group velocity of the $\mathrm{n}^{\text {th }}$ mode is $V_{n}=d \omega_{n} / d k$.

TABLE I. The constants and parameters used to calculate the phonon thermal conductivity of PVAc (see Refs. 35 and 36).

\begin{tabular}{lc}
\hline \hline Parameters & Values \\
\hline$a$ & $5.2 \times 10^{8} \mathrm{~s}^{-1} \mathrm{~K}^{-1}$ \\
$b$ & $9.5 \times 10^{6} \mathrm{~s} \mathrm{~K}^{-4}$ \\
$A$ & $1.73 \times 10^{-28}$ \\
$B$ & $10^{12}$ \\
$h$ & $6.626 \times 10^{-34} \mathrm{~m}^{2} \mathrm{~kg} \mathrm{~s}^{-1}$ \\
$k_{B}$ & $1.38 \times 10^{-23} \mathrm{~m}^{2} \mathrm{~kg} \mathrm{~s}^{-2} \mathrm{~K}^{-1}$ \\
$T_{1}$ & $0.4 \mathrm{~K}$ \\
$T_{2}$ & $10 \mathrm{~K}$ \\
$V_{0}$ & $2 \times 10^{-6} \mathrm{~m}^{3}$ \\
$\rho$ & $1.19 \times 10^{3} \mathrm{~kg} \mathrm{~m}^{-3}$ \\
$\omega_{D}$ & $0.8 \times 10^{13} \mathrm{~Hz}$ \\
\hline \hline
\end{tabular}

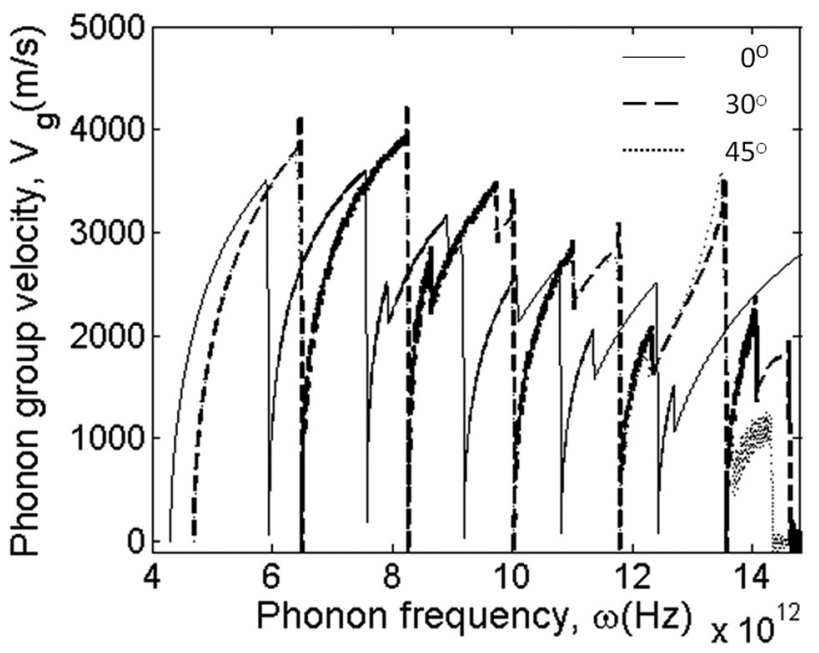

FIG. 3. Phonon group velocity versus phonon frequency for a $20 \mathrm{~nm}$ diameter PVAc nanofiber experiencing different angles of twist.

For both twisted and untwisted cylinders, the phonon velocity is pulselike. This velocity shifts towards larger frequencies when twist is applied. At the same frequency, phonons in an untwisted nanofiber travel at a higher speed than do phonons in the twisted nanofiber. Therefore, twisting leads to phonon blockage, which reduces the velocity. Figure 4 presents the effect of twisting on the phonon group velocity where data from Fig. 3 and from other angles of twist are averaged over various frequencies. Twisting is an obstacle to phonon transport since it reduces the phonon group velocity. There is a rapid decrease in this velocity for larger twist angles, particularly for angles greater than $45^{\circ}$.

In order to determine the phonon mean free path in a non-crystalline structure, we use the method of Walton and Dubey et $a l .{ }^{33-35}$ where the combined scattering relaxation rate is expressed by Eq. (17). The material constants used in our simulation are provided in Table I. Figure 5 presents the phonon thermal conductivity of a twisted nanofiber for different angles of twist. Solid dots represent experimental measurements ${ }^{35,36}$ for untwisted PVAc in the $0.1-4 \mathrm{~K}$ range as shown in the inset, while our simulations cover higher temperatures until $320 \mathrm{~K}$. The simulations agree well with the experimental data at very low temperatures. At these temperatures, the number of thermally excited phonons is relatively small so that they are no longer important during

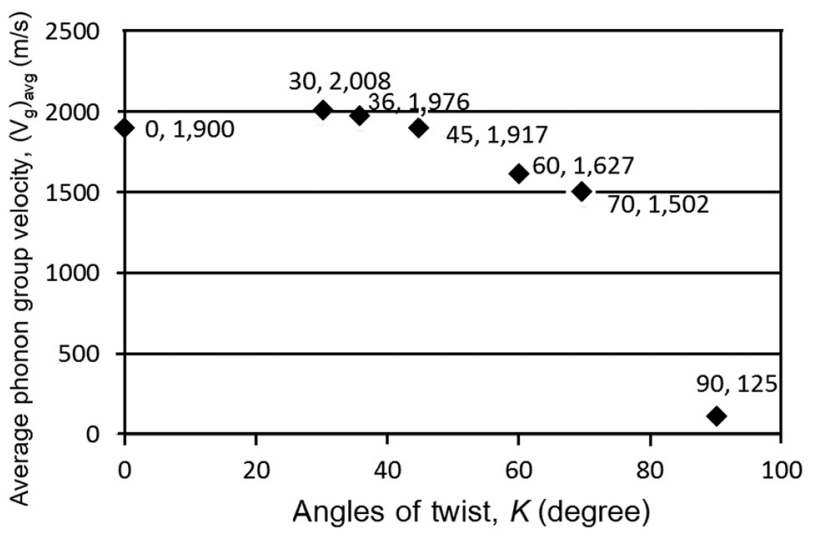

FIG. 4. Average phonon group velocity as a function of angles of twist for a $20 \mathrm{~nm}$ diameter PVAc nanofiber. 


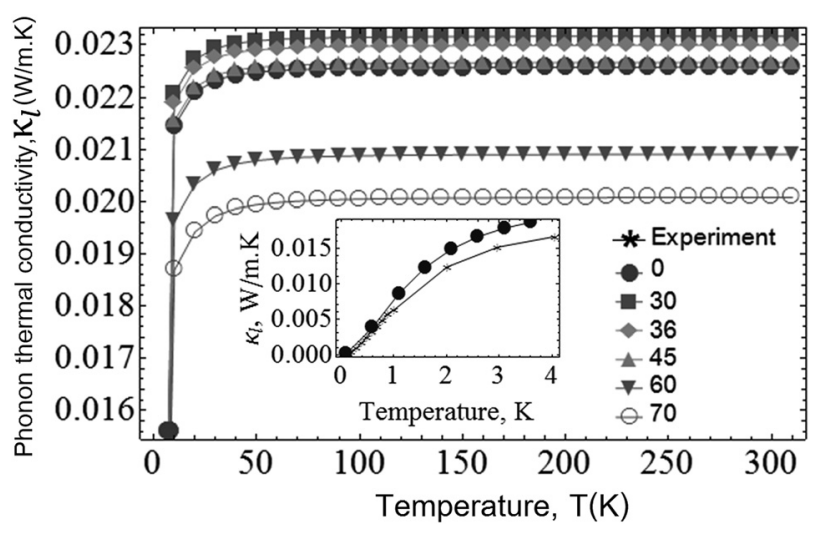

FIG. 5. The variation in phonon thermal conductivity for a $20 \mathrm{~nm}$ diameter PVAc nanofiber at different angles of twist as a function of temperature.

scattering. Rather, phonons are scattered by crystal defects or boundaries. The more a nanofiber is twisted, the more helical are its atom chains. Atomic vibrations in such a deformed structure have a shorter mean free path, which enhances scattering at the multiple interfaces that are produced through twisting, leading to a lower thermal conductivity. Therefore, the deformation of the interfaces due to twisting has a strong influence on the lattice thermal conductivity at lower temperatures. From Fig. 5, we note that the conductivity remains almost unchanged up to $K=45^{\circ}$ after which it decreases by about $7 \%$ from its initial value at $300 \mathrm{~K}$ for an untwisted nanofiber. The conductivity abruptly decreases from $0.0226 \mathrm{~W} \mathrm{~m}^{-1} \mathrm{~K}^{-1}$ for an angle of $45^{\circ}$ to $0.020 \mathrm{~W} \mathrm{~m}^{-1} \mathrm{~K}^{-1}$ when the angle is $70^{\circ}$, a change of $\approx 13 \%$.

For the larger simulated temperature range, the contribution from phonon scattering at frequencies in the range of $\left(\omega_{p t}-\omega_{D}\right)$ dominates. It is inversely proportional to the phonon group wave velocity. Because the number of phonons increases with increasing temperature, phonon-phonon scattering becomes important, which decreases the phonon mean free path. However, due to an abrupt decrease in $\left(V_{g}\right)_{a v g}$ at twisting angles larger than $70^{\circ}$, there is an associated significant increase in $\tau_{A P}$ so that, consequently, $\kappa_{A P}$ increases. Therefore, the total thermal conductivity of a nanofiber subjected to a very large angle of twist increases. As discussed above, a twisted nanofiber has both improved mechanical properties and electrical conductivity. We have shown that a deformed shape influences the phonon thermal conductivity which it can guide material design and selection.

Mechanical stress can tailor phonon transport and thus heat removal by modifying phonon dispersion, which could be useful for thermoelectric applications and the thermal management of nanodevices. We focus on torsional effects on phonon transport and the resulting thermal conductivity by theoretically investigating the propagation of elastic waves in an initially twisted PVAc nanofiber using Mooney's model based on the wave equation that accounts for different angles of twist. Phonons in an untwisted nanofiber use less energy to travel at the same speed than do phonons in a twisted fiber. Phonon dispersion in an untwisted nanofiber is different from that for its twisted counterpart in that each mode displays lower energies. Twisting is an obstacle to phonon transport, which reduces the phonon group velocity, hence altering the lattice thermal conductivity. Twist angles between $45^{\circ}$ and $70^{\circ}$ produce deformations that reduce the phonon thermal conductivity, thus, enhancing the figure of merit in thermoelectrics. However, when $K>70^{\circ}$, phonon frequencies larger than the plateau frequency increase, thus, increasing the conductivity. Hence, phonon engineering can be used to tune the phonon thermal conductivity.

This work was supported by National Nanotechnology Center (NANOTEC). M. Liangruksa acknowledges the useful discussions with SIM-lab (Nanoscale Simulation Laboratory) members at NANOTEC.

${ }^{1}$ Y. Hasegawa, M. Murata, D. Nakamura, and T. Komine, J. Appl. Phys. 106(6), 063703 (2009).

${ }^{2}$ G. J. Snyder and E. S. Toberer, Nature Mater. 7(2), 105-114 (2008).

${ }^{3}$ A. Balandin and K. L. Wang, J. Appl. Phys. 84(11), 6149-6153 (1998).

${ }^{4}$ L. Weber and E. Gmelin, Appl. Phys. A: Mater. Sci. Process. 53(2), 136-140 (1991).

${ }^{5}$ R. Kim, S. Datta, and M. S. Lundstrom, J. Appl. Phys. 105(3), 034506 (2009).

${ }^{6}$ F. Song, G. L. Huang, and V. K. Varadan, Acta Mech. 209(1-2), 129-139 (2010).

${ }^{7}$ A. Majumdar, Science 303(5659), 777-778 (2004).

${ }^{8}$ R. Zuzok, A. B. Kaiser, W. Pukacki, and S. Roth, J. Chem. Phys. 95(2), 1270-1275 (1991).

${ }^{9}$ Y. W. Park, Synth. Met. 45(2), 173-182 (1991).

${ }^{10}$ N. T. Kemp, A. B. Kaiser, C. J. Liu, B. Chapman, O. Mercier, A. M. Carr, H. J. Trodahl, R. G. Buckley, A. C. Partridge, J. Y. Lee, C. Y. Kim, A. Bartl, L. Dunsch, W. T. Smith, and J. S. Shapiro, J. Polym. Sci. Part B: Polym. Phys. 37(9), 953-960 (1999).

${ }^{11}$ N. Mateeva, H. Niculescu, J. Schlenoff, and L. R. Testardi, J. Appl. Phys. 83(6), 3111-3117 (1998).

${ }^{12}$ N. Toshima, Macromol. Symp. 186, 81-86 (2002).

${ }^{13}$ J. E. Österholm, P. Passiniemi, H. Isotalo, and H. Stubb, Synth. Met. 18(1-3), 213-218 (1987).

${ }^{14}$ I. Lévesque, P.-O. Bertrand, N. Blouin, M. Leclerc, S. Zecchin, G. Zotti, C. I. Ratcliffe, D. D. Klug, X. Gao, F. Gao, and J. S. Tse, Chem. Mater. 19(8), 2128-2138 (2007).

${ }^{15}$ Q. B. Pei, G. Zuccarello, M. Ahlskog, and O. Inganas, Polymer 35(7), 1347-1351 (1994).

${ }^{16}$ B. L. Groenendaal, F. Jonas, D. Freitag, H. Pielartzik, and J. R. Reynolds, Adv. Mater. 12(7), 481-494 (2000).

${ }^{17}$ L. Groenendaal, G. Zotti, and F. Jonas, Synth. Met. 118(1-3), 105-109 (2001).

${ }^{18}$ N. Koch, A. Vollmer, and A. Elschner, Appl. Phys. Lett. 90(4), 043512 (2007).

${ }^{19}$ M. H. Bolin, K. Svennersten, X. J. Wang, I. S. Chronakis, A. RichterDahlfors, E. W. H. Jager, and M. Berggren, Sens. Actuators, B 142(2), 451-456 (2009).

${ }^{20}$ C. Yu, K. Choi, L. Yin, and J. C. Grunlan, ACS Nano 5(10), 7885-7892 (2011).

${ }^{21}$ C. Yu, Y. S. Kim, D. Kim, and J. C. Grunlan, Nano Lett. 8(12), 4428-4432 (2008).

${ }^{22}$ D. Kim, Y. Kim, K. Choi, J. C. Grunlan, and C. H. Yu, ACS Nano 4(1), 513-523 (2010).

${ }^{23}$ Y. Ryu, L. Yin, and C. H. Yu, J. Mater. Chem. 22(14), 6959-6964 (2012).

${ }^{24}$ D. D. Freeman, K. Choi, and C. Yu, Plos One 7(11), e47822 (2012).

${ }^{25}$ A. Balandin and K. L. Wang, Phys. Rev. B 58(3), 1544-1549 (1998).

${ }^{26}$ M. J. Huang, W. Y. Chong, and T. M. Chang, J. Appl. Phys. 99(11), 114318 (2006).

${ }^{27}$ A. Khitun, A. Balandin, and K. L. Wang, Superlattices Microstruct. 26(3), 181-193 (1999).

${ }^{28}$ A. Khitun and K. L. Wang, Appl. Phys. Lett. 79(6), 851-853 (2001).

${ }^{29}$ X. Lu, J. H. Chu, and W. Z. Shen, J. Appl. Phys. 93(2), 1219-1229 (2003).

${ }^{30}$ A. Pevzner, Y. Engel, R. Elnathan, A. Tsukernik, Z. Barkay, and F. Patolsky, Nano Lett. 12(1), 7-12 (2012).

${ }^{31}$ M. Liangruksa and I. K. Puri, J. Appl. Phys. 109(11), 113501 (2011).

${ }^{32}$ H. Demiray and E. S. Şuhubi, Int. J. Eng. Sci. 8(1), 19-30 (1970).

${ }^{33}$ D. Walton, Solid State Commun. 14(4), 335-339 (1974).

${ }^{34}$ A. F. Saleh and K. S. Dubey, Acta Phys. 51(3), 265-271 (1981).

${ }^{35}$ A. K. Hasen and K. S. Dubey, Solid State Commun. 38(12), 1185-1187 (1981).

${ }^{36}$ C. M. Roland and R. Casalini, Macromolecules 36(4), 1361-1367 (2003). 